\title{
Ultrasound signs in the diagnosis of placental anomalies: placenta accreta at the level of the uterine scar
}

\author{
Oana-Denisa Bălălău1,2, Adina-Teodora Corbu1,2, Cristian Bălălău², Romina-Marina \\ Sima ${ }^{1,2}$, Liana Pleș ${ }^{1,2}$, Anca-Daniela Stănescu ${ }^{1,2}$
}

${ }^{1}$ St. Ioan Clinical Emergency Hospital - Bucur Maternity, Bucharest, Romania

${ }^{2}$ Carol Davila University of Medicine and Pharmacy, Bucharest, Romania

\begin{abstract}
Introduction. The incidence of Caesarian section has increased remarkably in recent years, which also leads to the increased maternal and fetal morbidity due to the complications that occur after the changes in the myometrial structure of the uterine scar. Placental anomalies with an increased risk for both the mother and the fetus are known under the heading of AIP (abnormally invasive placentation). Approximately $80 \%$ of the cases of placental anomalies are associated with a history of Caesarian delivery, curettage or myomectomies with the opening of the uterine cavity.

Aims and scope. The present paper aims at highlighting the current techniques of diagnosing placental abnormalities from the first trimester of pregnancy. Several studies in the specialty literature have been evaluated, those published in the last 5 years (2015-2019) on the PubMed platform, the results being presented in the form of a review.

Results. In 2018, Pagani et al. conducted a meta-analysis on 20 studies in the specialty literature. The current techniques used to diagnose placental abnormalities are abdominal and transvaginal ultrasound and Doppler ultrasound. A number of 7 studies (721 cases) used ultrasound to diagnose abnormalities. The identification of the degree of invasion was performed with a specificity of $97.1 \%$ - placenta accreta, $98.4 \%$ - placenta increta, $98.9 \%$ - placenta percreta and a sensitivity of $90.6 \%$, $93 \%$ and $81.2 \%$ respectively. The main signs evidenced upon ultrasound, which are currently used by most authors in specialized studies are: the loss/ the interruption of the hypoechoic area at the level of the myometrium under the placental bed, placental gaps (which may exhibit turbulent blood flow), the interruption of the hyperechoic line represented by the posterior bladder wall and the uterine serous membrane at this level, the thinning of the myometrium (reaching $<1 \mathrm{~mm}$ ), the presence of an exophytic formation which exceeds the uterine serous membrane, infiltrating into the urinary bladder. The echo Doppler images reveal: blood flow at the level of the placental gaps, intense vascularization in the subplacental area and vesicouterine hyper-vascularization. In a paper published in 2018, Cali et al. highlighted the importance of identifying these ultrasound signs from the first trimester of pregnancy. The accuracy of diagnosing AIP in the first trimester is low compared to the $2^{\text {nd }}$ and $3^{\text {rd }}$ trimesters: the loss of the hypoechoic area $84.3 \%$ vs. $92.4 \%$, the presence of placental gaps $78.3 \%$ vs. $100 \%$, the interruption of the hyperechoic line at the level of the urinary bladder $75.9 \%$ vs. $93.3 \%$, vesicouterine hyper-vascularization $50.6 \%$ vs. $81 \%$.

Conclusions. The importance of making an accurate diagnosis, as early as possible, of a placental anomaly is vital. The close monitoring of the pregnant woman, with serial ultrasounds performed quarterly, which should follow the signs indicated until so far in the specialized studies, as well as the preparation of a multidisciplinary operative team increases the probability of giving birth to a living new born baby, as well as the chance of preserving maternal fertility.
\end{abstract}

Keywords

: placenta accreta, placental anomalies, uterine scars, diagnosis, ultrasonography

Highlights

$\checkmark$ The diagnosis of placental malformation from the first trimester can decrease maternal morbidity by reducing the hemorrhagic complications and by preparing the management of delivery beforehand.

To cite this article: Bălălău $\mathrm{OD}$, Corbu AT, Bălălău C, Sima RM, Pleș L, Stănescu AD. Ultrasound signs in the diagnosis of placental anomalies: placenta accreta at the level of the uterine scar. J Clin Invest Surg. 2019; 4(2): 77-80. DOI: 10.25083/2559.5555/4.2/77.80 


\section{Introduction}

Placental abnormalities with an increased risk for both the mother and the fetus come under the heading of AIP (abnormally invasive placentation). These result from the implantation of the placenta in an area with defective decidualization, scar tissue, as in the case of the myometrium in the area of the post-Caesarian section scar. Thus, the placenta is directly implanted into the myometrium which it can actually penetrate and reach the serous uterine membrane (1).

The process of decidualization is influenced by various molecules such as: growth factors, cytokines, adhesion molecules, hormones and enzymes. Natural Killer cells have proven an important role in the trophoblastic invasion. In his study, Leban et al. highlighted a small number of NK cells in the cases of placenta accreta through immunohistochemical tests (2).

The term AIP was introduced in 2013 and it refers to the impossibility of spontaneously or manually removing the placenta from the site of insertion without causing severe bleeding.

Other theories regarding the pathogenesis of placental abnormalities are also described in the specialty literature, such as: poor vascular remodeling in the area of uterine scar or microscopic endometrial defects in the case of submucosal fibromas, adenomyosis.

The incidence of Caesarian section has remarkably increased in recent years, which also leads to the increase of maternal and fetal morbidities, due to the complications that occur after the changes in the myometrium at the level of the uterine scar (3). Approximately $80 \%$ of the cases of placental abnormalities are associated with a history of Caesarian delivery, curettages or myomectomies with the opening of the uterine cavity. The pregnancy inserted or developed at the level of the post Caesarian section scar is also a precursor of abnormal placental adherence (4).

The importance of the imaging techniques for the early diagnosis of placental abnormalities has been proven by the lower incidence of peripartum complications.

\section{Discussions}

The present paper aims at highlighting the current techniques for diagnosing placental abnormalities from the first trimester of pregnancy, with emphasis of the ultrasound techniques. The studies in the specialty literature published in the last years (2015-2019) on PubMed platform were evaluated, the results being presented in the form of a review.

The analysis of the literature studies has revealed an increased rate in the diagnosis of placental abnormalities through ultrasounds and MRI. A series of indices and ultrasound and color Doppler signs have been revealed.

Maternal mortality associated with placenta accretatype abnormalities is $10 \%$, while morbidity is $75 \%$ due to complications such as: uterine rupture, massive bleeding, necessity hysterectomy (5). The number of Caesarian sections in the patient's history increases proportionally with the risk of placental abnormalities: a history of Caesarian section - a $3 \%$ risk vs. a history of more than 3 Caesarian sections - a $40 \%$ risk.

In 2018, Pagani et al. conducted a meta-analysis on 20 studies in the specialty literature, which included 3,209 pregnant women at risk of AIP (the main risk factor being considered the association between placenta praevia and a history of Caesarian section or surgery on the uterus). A number of 407 pregnant women were diagnosed with AIP: $37.8 \%$ - placenta accreta, $32.2 \%$ - placenta increta, $30 \%$ placenta percreta. The current techniques used in diagnosing the placental abnormalities are abdominal or transvaginal ultrasound and Doppler ultrasound. There are studies that demonstrate the usefulness of MRI scans, more precisely establishing the topography and the extent of the placental invasion.

A number of 7 studies (721 cases) used ultrasound for the diagnosis of anomalies and the determination of the degree of placental invasion. The identification of the degree of invasion was achieved with a specificity of $97.1 \%$ - placenta accreta, $98.4 \%$ - placenta increta, $98.9 \%$ - placenta percreta and a sensitivity of $90.6 \%, 93 \%$ and $81.2 \%$ respectively (3).

The main ultrasound signs highlighted, which are currently used by most authors in their specialty studies, are: the loss/ the interruption of the hypoechoic area at the level of the myometrium under the placental bed, placental gaps (large, irregular, transonic spaces that can present turbulent blood flow), the interruption of the hyperechoic line represented by the posterior vesical wall and the uterine serous membrane at this level, the thinning of the myometrium (reaching $<1 \mathrm{~mm}$ ), the presence of a exophytic area which exceeds the uterine serous membrane, infiltrating the urinary bladder. The echo Doppler images reveal: blood flow at the level of the placental gaps, most often exhibiting a turbulent character, the intense vascularization in the subplacental area and the hyper-vascularization of the vesicouterine interface $(3,5$ 7).

In his paper published in 2018, Cali et al. discussed 188 cases that presented risk factors of placental anomalies and highlighted the importance of identifying these ultrasound signs from the first trimester of pregnancy. The authors 
state that up to $90 \%$ of the cases of AIP can be detected in the first trimester. The accuracy of diagnosing AIP in the first trimester is low compared to the 2 nd and the $3 \mathrm{rd}$ trimester: the loss of the hypoechoic area $84.3 \%$ vs. $92.4 \%$, the presence of placental gaps $78.3 \%$ vs. $100 \%$, the interruption of the hyperechoic line at the level of the urinary bladder $75.9 \%$ vs. $93.3 \%$, vesicouterine hypervascularization $50.6 \%$ vs. $81 \%$ (8). A specificity of $100 \%$ and a sensitivity of $78.3 \%$ were achieved for the presence of placental gaps along with the loss of the hypoechoic area and the same specificity and a similar sensitivity (75.9\%) for the interruption of the vesical wall along with the loss of the hypoechoic area. These combinations of ultrasound signs were evaluated at 11-14 weeks of pregnancy.

Comslock et al. conducted a prospective study over a period of 12 years on 163,000 female patients. Out of these, 2,002 presented risk factors for the development of placental anomalies: a history of placenta praevia and Caesarian section, while 33 had ultrasound signs suspicious of placenta accreta. The placental gaps were highlighted with a sensitivity of $93 \%$ (9).

J. Yang et al. introduced in their study a number of 51 patients with total placenta praevia and a history of Caesarian delivery and made a classification of the degrees of intraplacental gaps: degree 0 - no gap, degree 1 between 1 and 3 gaps, degree 2 - between 4 and 6 gaps and degree 3 - multiple large gaps and with irregular layout. In the cases classified as being greater than or equal to degree 1 , the method used exhibited a sensitivity of $86.7 \%$ and a specificity of $78.6 \%$ in the diagnosis of placental anomalies, while in the case of at least degree 2 classification - a sensitivity of $100 \%$ and a specificity of 97.2\% were achieved (10).

Another classification system for predicting placental anomalies, using ultrasonography and color Doppler ultrasounds, was the objective of J. Tobivin et al. in a paper published in 2018, on 258 female patients. They granted points from 1-12 according to the following criteria: the number ( $<=2-1$ point, $>2-2$ points) and the size of placental gaps ( $<=2 \mathrm{~cm}-1$ point, $>2 \mathrm{~cm}-2$ points), the lack of utero-placental demarcation ( 2 points), the number of Caesarian sections in the patient's history $(1-1$ point, >=2 -2 points), the location of the placenta (anterior -1 point, praevia -2 points), blood flow at the level of the gaps (1 point), utero-placental hyper-vascularization (2 points). The classification was divided in three categories: less than 5 points - low risk, between $6-7$ points - moderate risk, between $8-12$ points - high risk. 23 of the patients had an adherent placenta, and $84.2 \%$ of them were included in the high risk class, thus requiring hysterectomy (with a $100 \%$ histopathological confirmation of the placental abnormality) (11).

Besides the ultrasound diagnostic signs, there are certain MRI indices that are used when placental abnormalities are suspected, with low sensitivity and specificity ( $80-88.8 \%$ and $65-86.6 \%$ respectively) (1214). In 2017, A. Valentini et al. published a study which followed the retrospective evaluation of the MRI images obtained during pregnancy (26-37 weeks of gestation).

A series of suggestive diagnostic signs was highlighted, but they failed in making a diagnosis of certainty (the high rate of false positive results in the case of highlighting a single MRI sign). Among these signs, there are: the thickness of the myometrium below $5 \mathrm{~mm}$ and the loss of the trilaminar structure, interrupted margins of the myometrium, heterogeneous intraplacental sign due to hemorrhage or vascular gaps, thick intraplacental bands in the T2 sequence with a diameter over $2 \mathrm{~cm}$, abnormal intraplancental vascularization, the loss of the pear-shaped form of the uterus, the direct visualization of the invasion into adjacent tissues, the loose layout of the urinary bladder, the placental protrusion into the internal cervical orifice (15). In order to accurately diagnose placental abnormalities by means of MRI, it is necessary to enrich the knowledge in the field and also to conduct more specialty studies.

\section{Conclusions}

The importance of making an accurate diagnosis, as early as possible, of some placental abnormalities is vital. The close monitoring of the pregnant woman, with serial ultrasounds, performed on a quarterly basis, which should follow the signs indicated until recently in the specialty studies, as well as the preparation of a multidisciplinary operative team increase the probability of delivering a healthy newborn baby, as well the chance of preserving maternal fertility. The diagnosis of placental malformation from the very first trimester can decrease maternal morbidity by reducing the hemorrhagic complications and by preparing the management of delivery beforehand.

\section{Conflict of interest disclosure}

There are no known conflicts of interest in the publication of this article. The manuscript was read and approved by all authors.

\section{Compliance with ethical standards}

Any aspect of the work covered in this manuscript has been conducted with the ethical approval of all relevant 
bodies and that such approvals are acknowledged within the manuscript.

\section{References}

1. Goh WA, Zalud I. Placenta accreta: diagnosis, management and the molecular biology of the morbidly adherent placenta. J Matern Fetal Neonatal Med. 2016; 29(11): 1795-800.

2. Laban M, Ibrahim EA-S, Elsafty MSE, Hassanin AS. Placenta accreta is associated with decreased decidual natural killer (dNK) cells population: a comparative pilot study. Eur J Obstet Gynecol Reprod Biol. 2014; 181: 284-8.

3. Pagani G, Cali G, Acharya G, Trisch I-T, PalaciosJaraquemada J, Familiari A, et al. Diagnostic accuracy of ultrasound in detecting the severity of abnormally invasive placentation: a systematic review and metaanalysis. Acta Obstet Gynecol Scand. 2018; 97(1): 2537. DOI: $10.1111 /$ aogs. 13238

4. Malik MF, Hoyos LR, Rodriguez-Kovacs J, Gillum J, Johnson SC. Placenta Increta Complicating Persistent Cesarean Scar Ectopic Pregnancy following Failed Excision with Subsequent Preterm Cesarean Hysterectomy. Case Rep Obstet Gynecol. 2016; 2016: 4071840. DOI: 10.1155/2016/4071840

5. Panaiotova J, Tokunaka M, Krajewska K, Zosmer N, Nicolaides KH. Screening for morbidly adherent placenta in early pregnancy. Ultrasound Obstet Gynecol. 2019; 53(1): 101-6.

6. Hsu C-C, Huang K-G. Evolving Cesarean Scar Pregnancy into Morbidity Adherent Placenta-Evidence from Serial Ultrasound Examination. J Med Ultrasound. 2017; 25(1): 47-51.

7. Fujisaki M, Furukawa S, Maki Y, Oohashi M, Doi K, Sameshima H. Maternal Morbidity in Women with Placenta Previa Managed with Prediction of Morbidly Adherent Placenta by Ultrasonography. J Pregnancy. 2017; 2017: 8318751. DOI: 10.1155/2017/8318751
8. Cali G, Forlani F, Foti F, Minneci G, Manzoli L, Flacco $\mathrm{ME}$, et al. Diagnostic accuracy of first-trimester ultrasound in detecting abnormally invasive placenta in high-risk women with placenta previa. Ultrasound Obstet Gynecol. 2018; 52(2): 258-64.

9. Comstock $\mathrm{CH}$, Love JJ, Bronsteen RA, Lee W, Vettraino IM, Huang RR, et al. Sonographic detection of placenta accreta in the second and third trimesters of pregnancy. Am J Obstet Gynecol. 2004; 190(4): 113540.

10. Yang JI, Lim YK, Kim HS, Chang KH, Lee JP, Ryu HS. Sonographic findings of placental lacunae and the prediction of adherent placenta in women with placenta previa totalis and prior Cesarean section. Ultrasound Obstet Gynecol. 2006; 28(2): 178-82.

11. Tovbin J, Melcer Y, Shor S, Pekar-Zlotin M, Mendlovic S, Svirsky R, et al. Prediction of morbidly adherent placenta using a scoring system. Ultrasound Obstet Gynecol. 2016; 48(4): 504-10.

12. Moretti F, Merziotis M, Ferraro ZM, Oppenheimer L, Fung Kee Fung K. The importance of a late first trimester placental sonogram in patients at risk of abnormal placentation. Case Rep Obstet Gynecol. 2014; 2014: 345348.

13. Maher MA, Abdelaziz A, Bazeed MF. Diagnostic accuracy of ultrasound and MRI in the prenatal diagnosis of placenta accreta. Acta Obstet Gynecol Scand. 2013; 92(9): 1017-22.

14. Kilcoyne A, Shenoy-Bhangle AS, Roberts DJ, Sisodia RC, Gervais DA, Lee SI. MRI of Placenta Accreta, Placenta Increta, and Placenta Percreta: Pearls and Pitfalls. Am J Roentgenol. 2017; 208(1): 214-21.

15. Valentini AL, Gui B, Ninivaggi V, Miccò M, Giuliani M, Russo L, et al. The morbidly adherent placenta: when and what association of signs can improve MRI diagnosis? Our experience. Diagn Interv Radiol. 2017; 23(3): 180-6. 